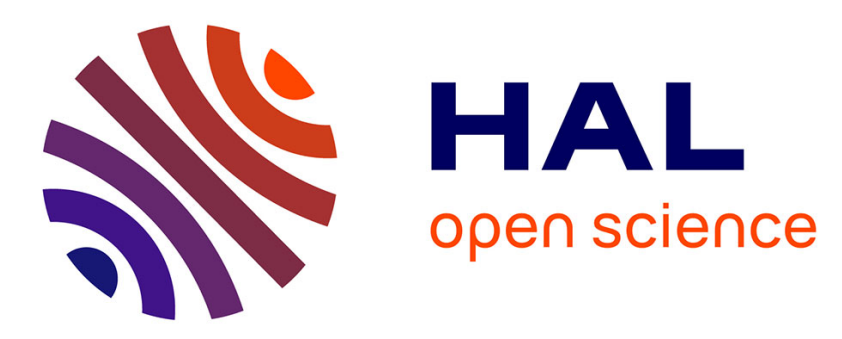

\title{
Magnetic anisotropy of one-dimensional Co nanostructures
}

\author{
Michel Daher Mansour, Romain Parret, F. Cheynis, Matthieu Petit, Fadi \\ Choueikani, Lisa Michez, Laurence Masson
}

\section{To cite this version:}

Michel Daher Mansour, Romain Parret, F. Cheynis, Matthieu Petit, Fadi Choueikani, et al.. Magnetic anisotropy of one-dimensional Co nanostructures. Physical Review B, 2020, 102 (15), pp.155403. 10.1103/PhysRevB.102.155403 . hal-02960045

\section{HAL Id: hal-02960045 https://hal-amu.archives-ouvertes.fr/hal-02960045}

Submitted on 15 Dec 2020

HAL is a multi-disciplinary open access archive for the deposit and dissemination of scientific research documents, whether they are published or not. The documents may come from teaching and research institutions in France or abroad, or from public or private research centers.
L'archive ouverte pluridisciplinaire HAL, est destinée au dépôt et à la diffusion de documents scientifiques de niveau recherche, publiés ou non, émanant des établissements d'enseignement et de recherche français ou étrangers, des laboratoires publics ou privés. 


\title{
Magnetic anisotropy of one-dimensional Co nanostructures
}

\author{
Michel Daher Mansour ${ }^{1}$, Romain Parret ${ }^{1}$, Fabien Cheynis ${ }^{1}$, Matthieu Petit ${ }^{1}$, Fadi Choueikani ${ }^{2}$, Lisa \\ Michez ${ }^{1}$ and Laurence Masson ${ }^{1, *}$ \\ ${ }^{1}$ Aix Marseille Univ, CNRS, CINaM, Campus de Luminy, Case 913, 13288 Marseille Cedex 9, France \\ ${ }^{2}$ Synchrotron SOLEIL, L'Orme des Merisiers, Saint-Aubin - BP 48, 91192 Gif-sur-Yvette Cedex, France \\ *Electronic mail: masson@cinam.univ-mrs.fr
}

\begin{abstract}
We report a combined scanning tunneling microscopy and $\mathrm{x}$-ray magnetic circular dichroism study to investigate the structural properties and magnetic behavior of a Co ultrathin film composed of dimer nanolines. These Co nanolines, $\sim 6 \mathrm{~nm}$ in length, are grown on a Si nanotemplate composed of nanoribbons self-organized on $\mathrm{Ag}(110)$. The first two Co layers present a weak magnetic response while upper Co layers exhibit an enhanced magnetization. Orbital and spin moments are experimentally determined. We show that an in-plane magnetization is favored and the magnetic anisotropy energy associated with the directions parallel and perpendicular to the nanolines are measured. The Co ultrathin film is shown to behave as a superparamagnetic system composed of onedimensional segments containing each $\sim 170$ ferromagnetically coupled Co atoms, with a blocking temperature estimated to be between $20 \mathrm{~K}$ and $40 \mathrm{~K}$. Our set of experiments allows for a comprehensive description of the magnetic behavior of the Co nanoline ultrathin film grown on a functionalized metallic substrate.
\end{abstract}

\section{INTRODUCTION}

Tremendous efforts have been devoted in the last two decades to investigate the magnetic properties of nanometer-size particles adsorbed on surfaces [1-4], motivated by both fundamental and practical interests such as magnetic recording or memory devices. An important attractive feature of these low-dimensional systems is the possibility to tune their magnetic properties by a fine control of their atomic structure or geometry and the chemical environment of the magnetic atoms. However, reducing the dimensionality of magnetic systems is known to reduce the stability of their magnetic ordering. One key parameter to describe the magnetic behavior of such nanoparticles is the magnetic anisotropy energy (MAE) which determines the tendency of the magnetization to align along specific axis rather than randomly fluctuate over time. Remarkably, transition metal (TM) nanostructures have been found to possess high magnetic anisotropy compared to two-dimensional (2D) ultrathin films or bulk materials, originating from the extremely low coordination of atoms [1]. Nevertheless, subnanometric nanostructures investigated are generally paramagnetic down to the lowest temperature, i.e. no remanent field is observed. In this context, one-dimensional (1D) TM nanostructures have received great attention since the discovery in 2002 of an unprecedented long-range ferromagnetic order in Co monoatomic chains formed at step edges of Pt(997), associated with the enhancement of 
Co orbital magnetic moments and, in turn, of MAE [5]. Such behavior results from kinetic barriers and below a threshold temperature, called blocking temperature ( $15 \mathrm{~K}$ in [5]), the MAE efficiently pins the orientation of the magnetization of each spin blocks along an easy axis without external magnetic field, on the timescale of the experiments. In these experiments, the average length of a continuous Co chains, uninterrupted by kinks, was $\sim 80$ Co atoms. At $45 \mathrm{~K}$, a short range ferromagnetic order, associated with a 1D superparamagnetic system, was found to extend over about 15 Co atoms only. This experimental pioneering work corroborates numerous theoretical calculations predicting that the reduced symmetry of 1D magnetic systems produces strong modifications of the electronic band structure and thus, significant effects on the MAE are expected [6-9]. The attractiveness of these 1D magnetic systems is reinforced with the recent theoretical prediction of an anisotropic spin-polarized electronic current along TM nanolines, such as Mn, Fe or Co nanolines on graphene [10], which opens the possibility of their integration in spintronic-based devices.

Another important feature of 1D magnetic nanostructures is an expected strong anisotropic behavior of the magnetization axis, determined by both the 1D geometry of the nanolines and their interaction with the substrate. Experimental studies have often revealed an uniaxial easy axis of magnetization in the plane perpendicularly to the TM chain axis, canted from the surface normal with different tilt angles $[5,11,12]$. Moreover, it has been shown that the easy axis of magnetization oscillates in the plane perpendicular to the chains axis as a function of the transverse width of Co chains grown on Pt(977) [13]. The different orientations of the easy axis can also originate from any changes in the environment as shown in several theoretical studies. For instance, a rotation of the easy axis from the direction parallel to the chain to the out-of-plane one has been theoretically predicted when cobalt chains are whether freestanding or supported on $\mathrm{Pd}(110)[6]$ while the magnetization of Co wires supported on $\mathrm{Cu}(100)$ and $\mathrm{Pt}(100)$ substrates was found to align with the wire axis [14].

Developments in atomic engineering during the last two decades have made possible the fabrication 1D TM systems with a high structural control over the width of the chains, allowing productive confrontations with theoretical works. Template directed-growth using intrinsic structural properties of surfaces has proven to be a powerful route to produce high densities of 1D nanostructures with controlled geometries, regular sizes and spacings, allowing the investigation of their magnetic properties using either local probes (spin-polarized scanning tunneling microscopy) or surface-average probes ( $x$-ray magnetic circular dichroism (XMCD) in the total electron yield (TEY) mode) $[5,11-13,15]$. Recently, we have shown that a nanopatterned $\operatorname{Ag}(110)$ surface with a $\mathrm{Si}$ nanoribbon (NR) array (density $\sim 5 \times 10^{6} \mathrm{~cm}^{-1}$ ) can be advantageously used to grow identical and highlyordered Co dimer nanolines [16]. Preliminary investigations of their magnetic properties using XMCD revealed that the first Co atomic layers directly adsorbed onto the Si NRs present a weak magnetic response (magnetic dead layer), while upper Co layers exhibit an enhanced magnetization with an inplane easy axis of magnetization, perpendicular to the Co nanolines.

In this paper, we have further investigated the magnetic properties of the ultrathin film composed of Co nanolines grown on the 1D Si nanotemplate. Advanced magnetic investigations using XMCD evidence a superparamagnetic behavior of thermally fluctuating $1 \mathrm{D}$ segments, with a low blocking temperature lying between $20 \mathrm{~K}$ and $40 \mathrm{~K}$ and the size of the spin blocks composed of 
ferromagnetically coupled Co atoms is estimated. Our results clearly evidence that an in-plane magnetization is favored. The magnetic moments together with the MAE associated with the directions parallel and perpendicular to the nanolines are measured.

\section{EXPERIMENT}

Growth and characterization measurements were performed in ultrahigh vacuum (UHV, base pressure $10^{-10}$ Torr) set-ups. The $\mathrm{Ag}(110)$ single-crystal surface was prepared in the UHV chamber by repeated cycles of $\mathrm{Ar}^{+}$sputtering followed by annealing at $770 \mathrm{~K}$. Si was evaporated from a direct current heated piece of silicon wafer kept at $1520 \mathrm{~K}$, after preoutgassing at $1070 \mathrm{~K}$ for several hours, onto the clean $\mathrm{Ag}(110)$ surface heated at $490 \mathrm{~K}$. The Ag surface nanopatterned with Si was checked by scanning tunneling microscopy (STM) and low energy electron diffraction (LEED) before the deposition of Co. Co was evaporated from a rod (purity $99.99 \%$ ) inserted in a commercial Scienta Omicron ebeam evaporator and was deposited on the Si NR template at $220 \mathrm{~K}$ prior to magnetic measurements. STM images were acquired at the CINaM in Marseille using Scienta Omicron scanning tunneling microscopes, working at room temperature (RT) and $77 \mathrm{~K}$ in constant current-mode. The STM data were processed using Gwyddion software [17]. XMCD experiments were performed the DEIMOS beamline at the French national synchrotron facility (SOLEIL), which operates in the soft x-ray range [18]. XMCD measurements were performed by measuring $x$-ray absorption spectra (XAS) at the Co $L_{2,3}$ edges ( $2 p$ to $3 d$ transitions) using left and right full circularly polarized light in the TEY mode. The magnetization curves were recorded using fast scan mode [19] at various substrate temperatures, ranging from $4 \mathrm{~K}$ to $100 \mathrm{~K}$, under a variable magnetic field of up to $6 \mathrm{~T}$, collinear with the incident $\mathrm{x}$-ray direction. The experiments performed with a magnetic field varying along or perpendicularly to the axis of the Co nanolines correspond to two different sets of experiments. The uncertainties associated with the measurements of the magnetic moments and the MAE result from XAS analysis. The Co coverages have been estimated using combined XAS and STM measurements. 1 monolayer of Co $\left(\mathrm{ML}_{\mathrm{Co}}\right)$ corresponds to the $5 \times 2 \mathrm{Si}$ NR array completely covered with Co nanolines and equals 0.6 monolayer (ML) in silver (110) surface atom density. The lattice parameters of $\mathrm{Ag}(110)$ are denoted $\mathrm{a}_{\mathrm{Ag} \mid \mathrm{I}}=0.289 \mathrm{~nm}$ in the [110] direction and $\mathrm{a}_{\mathrm{Ag} \perp}=0.409 \mathrm{~nm}$ in the [001] direction.

\section{RESULTS AND DISCUSSION}

\section{A. Growth of Co dimer nanolines on $\mathrm{Si} / \mathrm{Ag}(110)$}

Prior to Co deposition, the $\mathrm{Ag}(110)$ surface was firstly nanopatterned with a Si atomic layer, widely described in the literature (see for instance [20] and references therein, [21]). This nanotemplate consists in a Si NR array with a pitch of $\sim 2 \mathrm{~nm}$, formed during Si deposition at $490 \mathrm{~K}$ onto the bare $\mathrm{Ag}(110)$ surface. The $\mathrm{Si}$ NRs are self-assembled in a $5 \times 2$ superstructure covering uniformly the whole Ag surface at completion with a short- and long-range high structural order. The atomic structure of the Si NRs was solved in 2016 with an original Si pentamer chain model lying in the missing row troughs of the reconstructed $\mathrm{Ag}(110)$ surface $[20,22]$. In this description, Si NRs of the $5 \times 2$ superstructure correspond to twin Si pentamer chains and will be denoted hereafter as double 
nanoribbons (DNRs). The Si NR array is stable until $490 \mathrm{~K}$ [21], temperature above which a silver surface faceting induced by $\mathrm{Si}$ adatoms occurs [23].

It has been already reported in our group that this Si 1D nanotemplate can advantageously be used to grow Co nanolines [24,25]. It has been shown that upon Co deposition at RT, Co atoms are adsorbed on top of the Si NRs, giving rise to the growth of identical Co nanolines perfectly aligned with the NR axis. The high-resolution STM image of Fig. 3 published in [24] revealed that these Co nanolines of the first layer are composed of dimers oriented perpendicularly to the nanoline axis. Based on the accurate calibration of the STM image using the crystallographic atomic positions determined by grazing incidence x-ray diffraction (GIXD) (see [20] Supplemental Material) , the distance between two Co atoms of a dimer is $\mathrm{d}_{\mathrm{Co}-\mathrm{Co}_{\perp} \mathcal{L}}=(3.0 \pm 0.5) \AA$ and the distance between two adjacent Co atoms along the nanolines is higher, $\mathrm{d}_{\mathrm{co}-\mathrm{co} / /}=(4.5 \pm 0.5) \AA$ i.e $\sim 1.5 \mathrm{a}_{\mathrm{Ag} / \mid}$. This latter periodicity, in the direction parallel to the nanolines, leads to a modulation of the corrugation observed by STM: one out of every two dimers appears slightly brighter. Interestingly, STM images showed that after deposition of 0.16 $\mathrm{ML}$ of $\mathrm{Co}$ at RT, more than $80 \%$ of the dimer nanolines are side by side with two nanolines of similar length on the same Si DNR. Moreover, it has been shown that the length of Co nanolines is governed by the atomic process of Co inter-diffusion into the Si array, leading to the local destruction of the $\mathrm{Si}$ NRs [25]. To partially hinder this thermally activated process and thus increase the length of the Co nanolines, Co was deposited on the substrate held at $220 \mathrm{~K}$ prior to the XMCD measurements reported in the next section.

The STM image in Fig. 1(a) illustrates the growth of the first and second layers of Co nanolines on the $5 \times 2 \mathrm{Si}$ array, after deposition at $220 \mathrm{~K}$. The brighter protrusions correspond to Co dimers of the second layer while the other protrusions correspond to those of the first layer. Closer inspection of Fig. 1 (a) reveals the presence of some black holes, mostly located at the terminations of the Co nanolines and attributed to the local destruction of the Si NRs induced by Co diffusion into the Si layer, leaving uncovered parts of the silver substrate [25]. For both Co layers, the distance between the protrusions along the nanoline axis is $2 \mathrm{~d}_{\mathrm{co-co} / /}$, indicating that only protrusions with the higher corrugation of each layer are visible. Our STM observations suggest that the Co atomic arrangement in nanolines directly adsorbed on Si NRs and in the upper layers is similar. From STM images, we have analyzed the height of the Co nanolines measured from local bare parts of the silver substrate. As exemplified by the height profile displayed in Fig. 1(b), the corrugation of the first and second Co nanolines is found to be $\sim 50$ pm.

For higher coverages, the Co growth reproduces the 1D pattern of the Si template as shown in Fig. 2(a). By measuring the height of the different species observed in STM images, Co nanolines of the different layers can be identified, as illustrated in Fig. 2(b). The length of the nanolines cannot be accurately estimated due to the non-perfect layer-by-layer growth. Based on a statistical analysis of STM images, the average length at completion of the first Co layer grown at RT is $\sim 4.5 \mathrm{~nm}$. Assuming that the nanoline length of this first Co layer determines those of the following layers, the length of the Co nanolines of upper layers is $\sim 4.5 \mathrm{~nm}$, which is in agreement with the STM image of Fig. 2 (b). The average length at completion of the first Co layer grown at $220 \mathrm{~K}$ is $\sim 6 \mathrm{~nm}$, determining the length 
of the Co nanolines of the upper layers. We will consider in the following this length to be $6 \mathrm{~nm}$, which corresponds to nanolines composed of $\sim 14$ Co dimers.
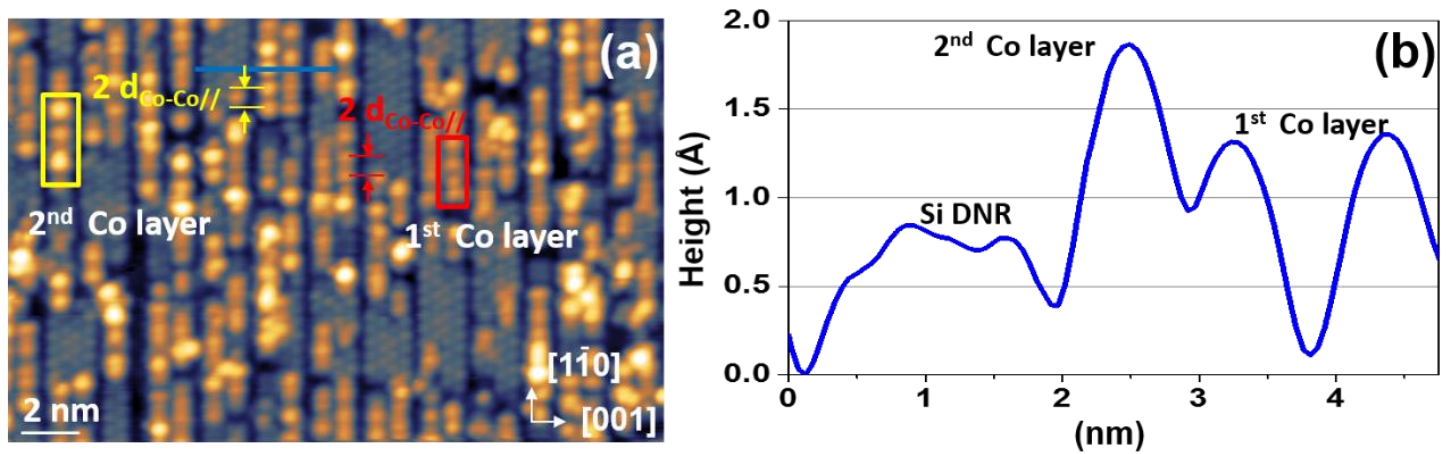

FIG. 1: (a) STM image of Co nanolines grown at $220 \mathrm{~K}$ on the $5 \times 2$ Si nanoribbon array. Co nanolines of the $1^{\text {st }}$ and $2^{\text {nd }}$ layers are indicated by colored rectangles (b) Height profile along the blue line in (a), showing typical corrugations associated with the different grown nanostructures on $\mathrm{Ag}(110)$.
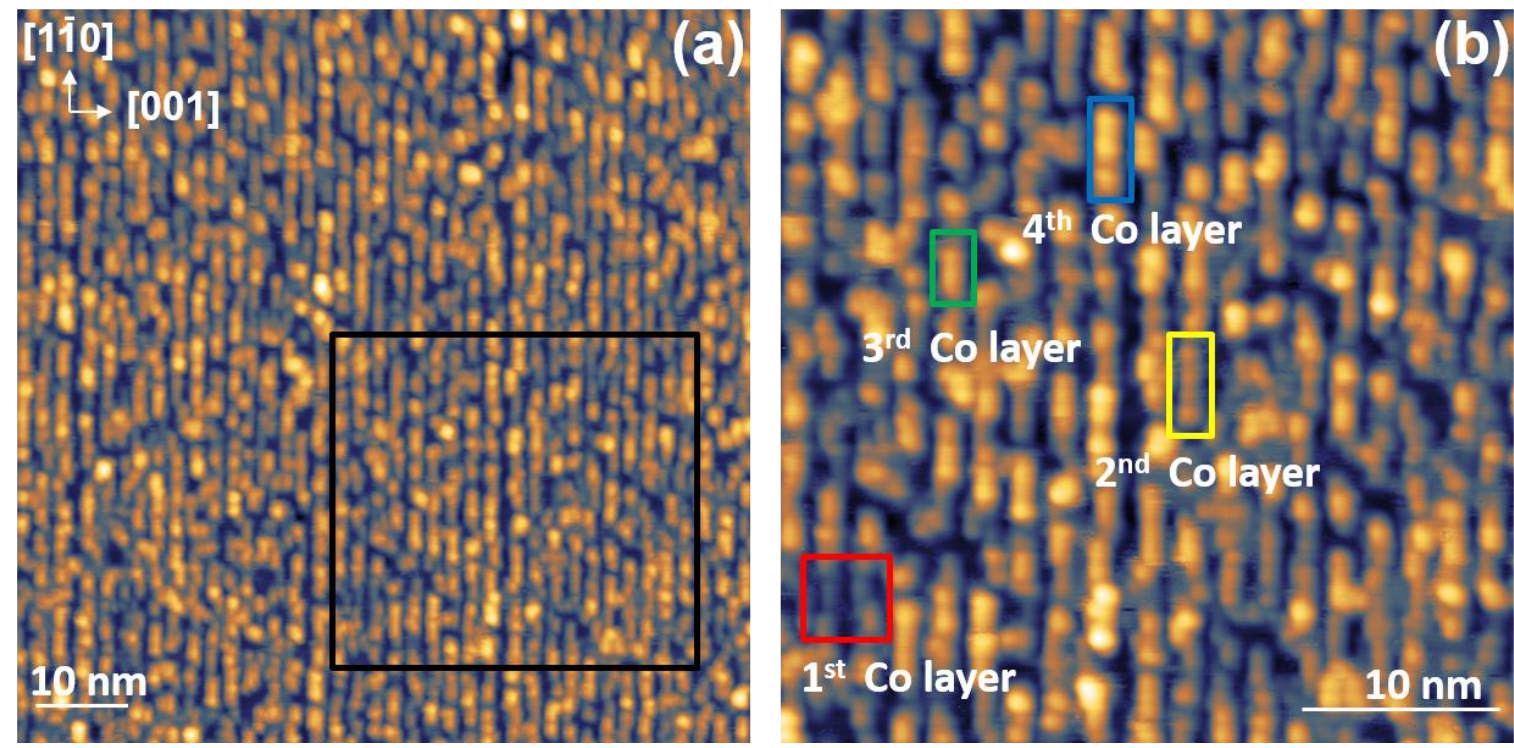

FIG. 2: STM images of an ultrathin film of Co nanolines (coverage $\leq 4 \mathrm{MLco}$ ) after deposition of Co at RT on the $5 \times 2 \mathrm{Si}$ array. (a) $100 \mathrm{~nm} \times 100 \mathrm{~nm}$. (b) Close view $(50 \mathrm{~nm} \times 50 \mathrm{~nm}$ ) of the area indicated in (a) by the black square box. Co nanolines of the different layers are indicated by colored rectangles.

\section{B. Magnetic properties of the Co dimer nanolines}

XAS spectra were recorded under a magnetic field of $6 \mathrm{~T}$ applied in the different geometries illustrated in Fig. 3(a), for right- and left-handed circularly polarized light denoted as $\mu_{+}$and $\mu_{-}$, respectively. $\mathrm{H}_{/ /}$and $\mathrm{H}_{\perp}$ denote a magnetic field parallel or perpendicular to the axis of the Co nanolines, respectively. To probe both the out-of-plane and the in-plane magnetic moments, the sample was rotated with respect to the direction of $\mathrm{H}_{/ /}$or $\mathrm{H}_{\perp}$ collinear to the x-ray beam, by an angle 
$\theta$ comprised between $0^{\circ}$ (normal incidence) and $70^{\circ}$ (close to grazing incidence). The strong nonmagnetic background signal coming from the Ag substrate has been removed from the Co- $L_{2,3}$ XAS spectra. The background for each considered temperature has been assessed as the difference between the XAS spectra recorded at the temperature of interest and $300 \mathrm{~K}$ where the Ag extended $\mathrm{X}$ ray absorption fine structure (EXAFS) oscillations are greatly attenuated. The spectra are normalized to the incident beam intensity and then to the $L_{3}$ pre-edge value. All the XAS spectra presented here have been recorded at the same Co coverage of $(4.0 \pm 0.5) \mathrm{ML}_{\mathrm{c}_{0}}$ i.e. an average of four layers of Co nanolines. This coverage has been estimated from XAS measurements at the $L_{2,3}$ Co absorption edges, taking into account the escape depth of electrons coming from the substrate through the adsorbate and the photoionization cross sections of both $\mathrm{Ag}$ and $\mathrm{Co}$ at the $L_{3} \mathrm{Co}$ absorption edge [26]. Height profiles measured in STM images recorded in situ on the samples probed by XAS are in agreement with this coverage. These STM images are very similar to the one presented in Fig. 2, with a mean thickness of 4 Co monolayers but with a lower resolution and thus not shown here.

Fig. 3(b) shows the XAS spectra at $4 \mathrm{~K}$ for both $\mathrm{x}$-ray polarizations and respectively $\mathrm{H}_{/ /}$(upper panel) and $\mathrm{H}_{\perp}$ (middle panel). The XAS spectra, that clearly show no trace of cobalt silicides, are characteristic of metallic Co [5]. Two broad absorption resonances are clearly visible at the $L_{3}$ and $L_{2}$ edges of $\mathrm{Co}$. The weak shoulder peak present at about $4 \mathrm{eV}$ above the $\mathrm{L}_{3}$ edge can be attributed to the presence of interface states for $\mathrm{Co}$ atoms located at the $\mathrm{Co} / \mathrm{Si}$ interface as suggested by Pong et al. [27]. The XAS spectra are similar for both orientations of the magnetic field. The XMCD signal that represents the difference between the XAS spectra for left- and right-handed light polarization gives access to the magnetization magnitude of a specific element. The spin $\left(m_{\mathrm{S}}\right)$ and orbital $\left(m_{L}\right)$ moments per atom can be quantitatively determined using the magnetic sum rules described in $[28,29]$. Here, we have applied the formalism described by Chen et al. [30], in order to evaluate the spin and orbital contributions to the magnetization of the Co nanolines. The number of holes in the Co $3 \mathrm{~d}$ band has been considered to be 2.5 , which corresponds to an average theoretical value for bulk Co [31,32], in agreement with the value of 2.4 used in the case of $\mathrm{Co}$ adatoms on $\mathrm{Pt}(111)$ [1]. For each configuration, the spin and orbital moments of the $4 \mathrm{ML}_{\mathrm{co}_{0}}$ ultrathin film derived from our measurements at grazing and normal incidences are similar, showing that the magnetization is saturated at $6 \mathrm{~T}$. We found $\mathrm{m}_{\mathrm{S} / /}=(0.51 \pm 0.06) \mu_{\mathrm{B}}$ per atom $\left(\mu_{\mathrm{B}} / \mathrm{at}\right)$ and $\mathrm{m}_{\llcorner/ /}=(0.073 \pm 0.010) \mu_{\mathrm{B}} /$ at for $\mathrm{H}_{/ /}$and $\mathrm{m}_{\mathrm{S} \perp}=(0.47 \pm 0.05) \mu_{\mathrm{B}} /$ at and $\mathrm{m}_{\llcorner}=(0.070 \pm$ 0.010) $\mu_{B} /$ at for $H_{\perp}$. The lower values of $m_{s \perp}$ and $m_{\perp}$ compared to those of $m_{s / /}$ and $m_{\llcorner/ /}$could result from a slightly lower Co coverage for the measurements performed in the $\mathrm{H}_{\perp}$ configuration, as revealed by the lower intensity in the XAS spectrum (see Fig. 3 (b)). Indeed, as already mentioned in [16] and confirmed below, the magnetic properties of the Co film depend on its thickness. To evaluate the real orbital and spin moments per atom, the weak contribution of the first two Co layers have been retrieved to the measurements reported above for $4 \mathrm{ML}_{c_{0}}$. For $2 \mathrm{ML}_{\mathrm{c}_{0}}$, the magnetic moments at normal incidence for the $\mathrm{H}_{/ /}$and $\mathrm{H}_{\perp}$ configurations are found to be similar and equal to $\mathrm{m}_{\mathrm{S}}=(0.09 \pm$ 0.01) $\mu_{B} /$ atom and $m_{L}=(0.012 \pm 0.002) \mu_{B} /$ atom. The moments of the Co atoms in the two upper layers of the $4 \mathrm{ML}_{C_{0}}$ film can thus be estimated to $\mathrm{m}_{\mathrm{S} / /}=(0.93 \pm 0.13) \mu_{\mathrm{B}} /$ at and $\mathrm{m}_{\mathrm{L} / /}=(0.134 \pm 0.022) \mu_{\mathrm{B}} /$ at in the $H_{/ /}$configuration. Similar estimations for the $H_{\perp}$ configuration lead to $m_{s \perp}=(0.85 \pm 0.11) \mu_{B} /$ at and $m_{\llcorner}=(0.128 \pm 0.022) \mu_{B} /$ at. These values are significantly lower than the bulk magnetic moments $\left(m_{S}\right.$ 
$=1.55 \mu_{B} /$ at and $m_{L}=0.153 \mu_{B} / a t$ ) [30]. We stress that a thicker Co film of nanolines should be deposited to recover bulk magnetic moments in the upper layers grown on the magnetic dead layer. These values are also significantly lower than the ones reported in our precedent work [16] for a 2 $\mathrm{ML}_{\mathrm{Co}_{0}}$ Co film ( $\mathrm{m}_{\mathrm{S}}=1.66 \mu_{\mathrm{B}} / \mathrm{at} ; \mathrm{m}_{\mathrm{L}}=0.20 \mu_{\mathrm{B}} /$ at). A closer inspection of the XAS spectra shown in Figs. $3(a)$ and $3(b)$ published in [16] reveals that the shoulder at $+4 \mathrm{eV}$ above the $\mathrm{L}_{3}$ edge is more pronounced in [16] than in the present work, suggesting that the chemical composition of the grown film or of the Si-Co interface was different in our previous study. The higher magnetic moments measured in [16] could result, for instance, from a less pronounced effect of the magnetic dead layer observed in the Co film.
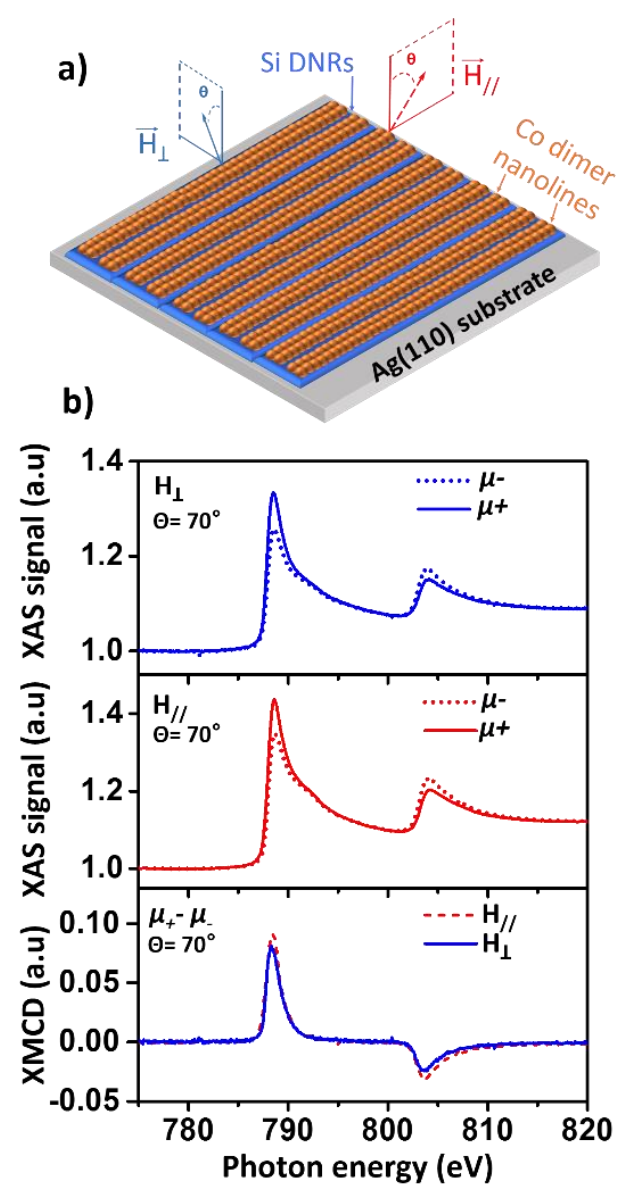

FIG. 3: (a) Schematic representation of the $H_{/ /}$and $H_{\perp}$ configurations for the XAS and XMCD measurements. For the angle dependant measurements, the sample was rotated by an angle $\theta$ relative to the surface normal, from normal $\left(\theta=0^{\circ}\right)$ to grazing incidence $\left(\theta= \pm 70^{\circ}\right)$. (b) XAS spectra for a Co coverage of $4 \mathrm{MLco}$, taken at grazing incidence $\left(\theta=70^{\circ}\right)$ at $4 \mathrm{~K}$ with a magnetic field of $6 \mathrm{~T}$ for both light helicities ( $\mu_{+}$and $\mu_{\text {-) }}$ ) and for $\mathrm{H}_{\perp}$ (upper panel) and $\mathrm{H}_{/ /}$(middle panel). The corresponding XMCD spectra are displayed in the lowest panel for both $\mathrm{H}_{/ /}$and $\mathrm{H}_{\perp}$ configurations.

Field-dependent magnetization curves, obtained from the XMCD signal for a coverage of 4 $\mathrm{ML}_{\mathrm{Co}}$, have been recorded at $4 \mathrm{~K}$ for different angles $\theta$ varying from normal incidence $\left(\theta=0^{\circ}\right)$ to grazing incidence $\left(\theta=70^{\circ}\right)$ in the $H_{/ /}$and $H_{\perp}$ configurations (see Fig. 4). No significant remanent magnetization 
at zero magnetic field was observed for both configurations, indicating the absence of long-range ferromagnetic order. However, the square shape of the magnetization curves suggests a short-range magnetic order and therefore an interatomic magnetic coupling. The angular dependence of the magnetization measured for an applied magnetic field of $0.25 \mathrm{~T}$ and normalized to the saturation value at $6 \mathrm{~T}$ is plotted in the inset of Figs. $4(\mathrm{a})$ and $4(\mathrm{~b})$ for the $\mathrm{H}_{/ /}$and $\mathrm{H}_{\perp}$ configurations, respectively. The results clearly evidence that an in-plane magnetization is favored. The MAE in the directions parallel and perpendicular to the nanolines can be derived from the magnetization curves along the easy and hard axes displayed in Figs. 4(a) and 4(b) using Eq. (2) in Ref. [33]. We obtain a MAE in the direction parallel to the Co nanolines : $K_{/ /}=(48 \pm 10) \mu \mathrm{eV} /$ at and a MAE in the direction perpendicular to the Co nanolines : $\mathrm{K}_{\perp}=(51 \pm 10) \mu \mathrm{eV} /$ at. These values are slightly lower than bulk h.c.p. Co $(70 \mu \mathrm{eV} / \mathrm{at})$. Surprisingly, the measured MAE is similar in both directions, suggesting an isotropic in-plane magnetization. Nevertheless, since strong in-plane anisotropic behavior of the magnetization has been evidenced theoretically and experimentally in similar 1D Co- and Fe-based systems [6, 8, 11, 14], such isotropic in-plane magnetization is not expected. We outline than no privileged in-plane orientations for the magnetization other than the direction parallel or perpendicular to the chains have been reported in all these studies. Interestingly, a theoretical study has shown that for Co monoatomic wires deposited on $\mathrm{Cu}(001)$, the easy axis is along the wires with a very small energy barrier between the directions parallel and perpendicular to the chain axis [14]. Similarly, a weak in-plane magnetic anisotropy between the axes parallel and perpendicular to the nanolines may be present, hidden in the uncertainty of our MAE measurements.
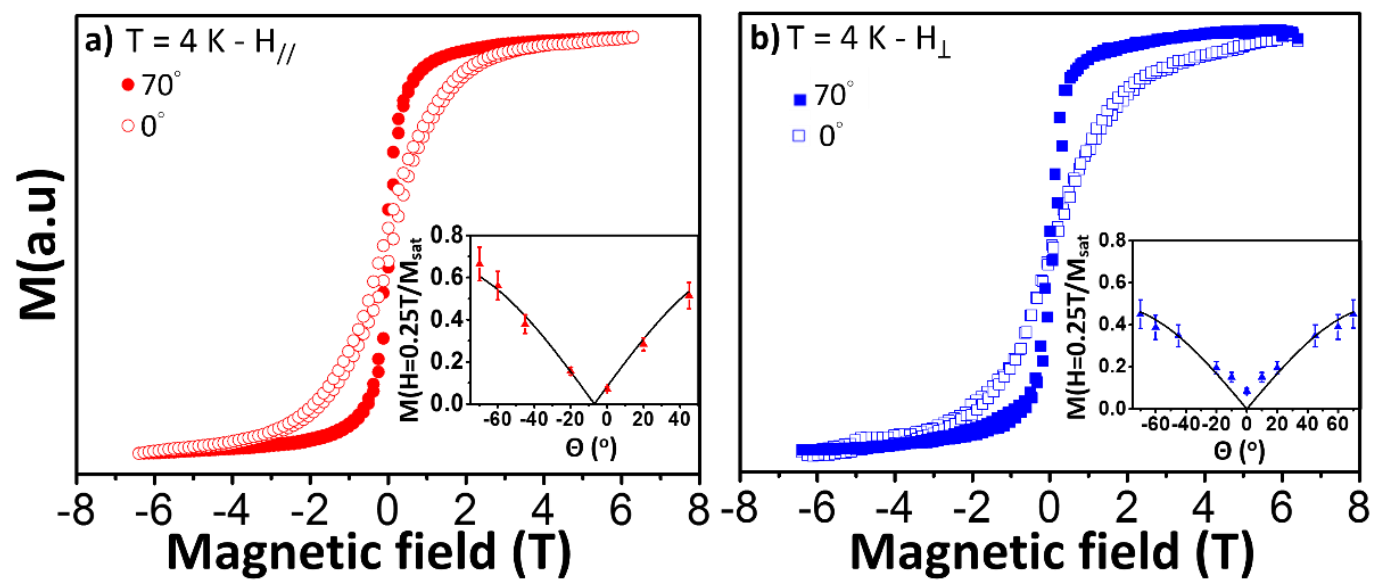

FIG. 4: Magnetization (M) of $4 \mathrm{MLco}$ recorded at $4 \mathrm{~K}$ at the $\mathrm{L}_{3}$ Co edge. $\mathrm{M}$ as a function of the applied field in the (a) $\mathrm{H}_{/ /}$configuration and (b) $\mathrm{H}_{\perp}$ configuration. Magnetization curves are measured at the grazing incidence $(\theta=$ $70^{\circ}$, filled red dots and filled blue squares) and at the normal incidence $\left(\theta=0^{\circ}\right.$, open red dots and open blue squares). The curves have been normalized to their saturation value at $6 \mathrm{~T}$. Insets: angular dependence of the magnetization. Variation in both configurations of the magnetization at $0.25 \mathrm{~T}$ normalized to the saturation magnetization at $6 \mathrm{~T}\left(\mathrm{M}_{\mathrm{sat}}\right)$ as a function of the incidence angle $\theta$. The solid lines are fits of the data according to the function $a\left|\sin \left(\theta-\theta_{0}\right)\right|$. 
To provide a comprehensive description of the magnetic behavior of our Co system, temperature-dependent magnetization measurements have been carried out. In Fig. 5, we have plotted the magnetization measured for an applied magnetic field $\mathrm{H}_{/ /}$and $\mathrm{H}_{\perp}$ of $0.25 \mathrm{~T}$ as a function of the temperature, after having applied a magnetic field of $6 \mathrm{~T}$. A plateau is observed below $20 \mathrm{~K}$ for both $H_{/ /}$and $H_{\perp}$ configurations, suggesting that a transition from a blocked state to a superparamagnetic configuration occurs above $20 \mathrm{~K}$. We can thus estimate that the blocking temperature $T_{B}$ is between $20 \mathrm{~K}$ and $40 \mathrm{~K}$.

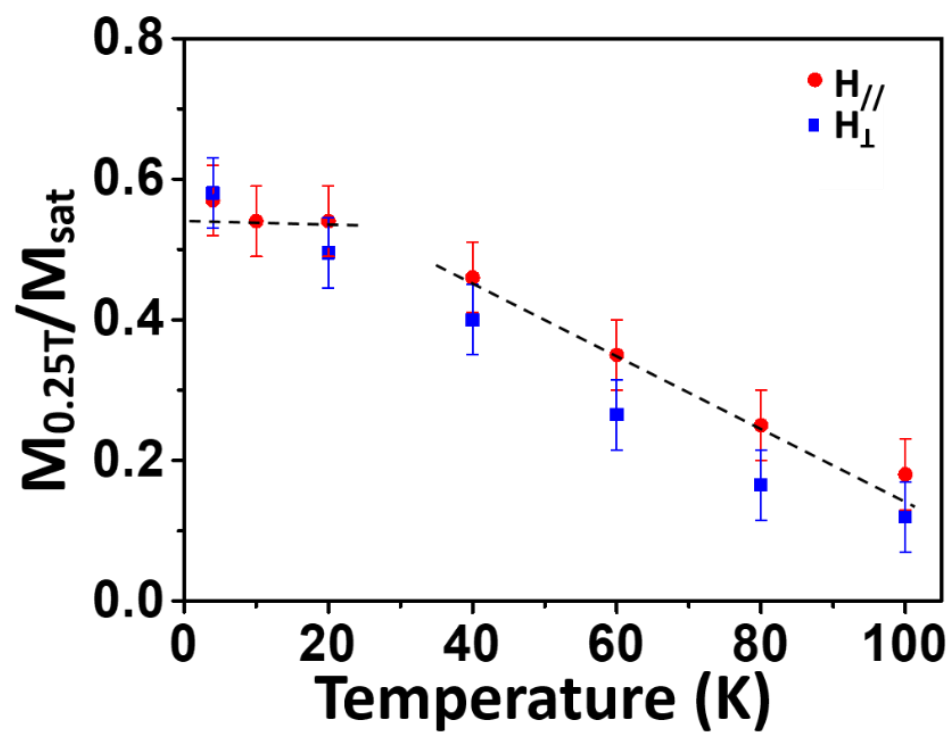

FIG. 5: Variation of the magnetization for an applied magnetic field of $0.25 \mathrm{~T}$ normalized to the saturation magnetization at $6 \mathrm{~T}\left(\mathrm{M}_{\text {sat }}\right)$ as a function of the temperature, for an applied magnetic field $\mathrm{H}_{/ /}$(filled red dots) and $\mathrm{H}_{\perp}$ (filled blue squares) at $\theta=70^{\circ}$. Black dashed lines are eye-guides for the blocked state and the superparamagnetic behavior.

To estimate the size of the spin blocks that compose the Co nanolines, we have studied the field-dependence of the magnetization as a function of the temperature in the $H_{/ /}$and $H_{\perp}$ configurations. In Figs. 6 (a) and 6(b), we present the magnetization curve of the $4 \mathrm{ML}_{\mathrm{co}}$ film recorded at $60 \mathrm{~K}$, measured along the two easy axes of magnetization, parallel and perpendicularly to the Co nanolines. In agreement with Fig. 5, the magnetization curve has a slightly more pronounced square

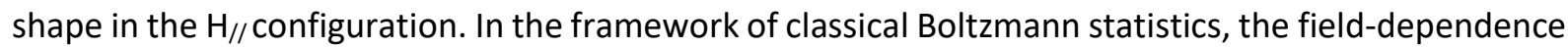
of the magnetization of an assembly of particles composed of $\mathrm{N}$ coupled atoms with magnetic moment $\mathrm{Nm}_{\text {tot }}$ and magnetic anisotropy NK obeys to:

$M=M_{\text {Sat }} \frac{\int \widehat{\boldsymbol{m}}_{\text {tot }} \cdot \widehat{\boldsymbol{H}} e^{\left(N \overrightarrow{\boldsymbol{m}}_{\text {tot }} \cdot \overrightarrow{\boldsymbol{H}}+N K\left(\widehat{\boldsymbol{m}}_{\text {tot }} \cdot \hat{\boldsymbol{e}}\right)^{2}\right) / k_{\mathrm{B}} T} d \Omega}{\int e^{\left(N \overrightarrow{\boldsymbol{m}}_{\text {tot }} \cdot \overrightarrow{\boldsymbol{H}}+N K\left(\widehat{\boldsymbol{m}}_{\mathrm{tot}} \cdot \hat{\boldsymbol{e}}\right)^{2}\right) / k_{\mathrm{B}} T} d \Omega}$

where $\mathrm{M}_{\mathrm{sat}}$ is the saturation value of the magnetization, $\widehat{\boldsymbol{e}}, \widehat{\boldsymbol{m}}_{\text {tot }}$, and $\widehat{\boldsymbol{H}}$ represent the unit vectors of the easy axis direction, the magnetic moment per atom and the field direction, respectively and $\overrightarrow{\boldsymbol{m}}_{\text {tot }}, \overrightarrow{\mathbf{H}}$ represent the vectors of the magnetic moment per atom and the applied field, respectively. 
The integration is carried out over the solid angle $\Omega$ of the magnetic moment in spherical coordinates. Considering that the upper Co layers are decoupled from the metallic silver substrate by the weakly magnetic first two Co layers, the total magnetic moments in both configurations of the two upper Co layers are given by $m_{\text {tot } / /}=m_{L / /}+m_{\mathrm{s} / /}$ and $m_{\text {tot } \perp}=m_{\perp \perp}+m_{\mathrm{S} \perp}$. The dashed lines in Figs. $6(a)$ and $6(b)$ represent the magnetization given by Eq. (1) with $\mathrm{N}=1$. We clearly see that the magnetic response of our Co system is different from the one expected for an assembly of isolated Co atoms. Thus, the Co nanolines behave more likely as a superparamagnetic system composed of segments (spin blocks) containing $\mathrm{N}$ exchange-coupled Co atoms. We define $\mathrm{N}_{/ /}$and $\mathrm{N}_{\perp}$ the number of coupled Co atoms derived from Eq. (1) with a magnetic field applied along and perpendicularly to the axis of the nanolines, respectively. By fitting with Eq. (1) the experimental magnetization curves shown in Figs. $6(\mathrm{a})$ and $6(\mathrm{~b})$ with the set of parameters $\left(\mathrm{m}_{\mathrm{tot} / /}=(1.06 \pm 0.15) \mu_{\mathrm{B}} / \mathrm{at} ; \mathrm{K}_{/ /}=(48 \pm 10) \mu \mathrm{eV} / \mathrm{at}\right)$ and $\left(\mathrm{m}_{\mathrm{tot} \perp}=\right.$ $\left.(0.98 \pm 0.13) \mu_{\mathrm{B}} / \mathrm{at}, \mathrm{K}_{\perp}=(51 \pm 10) \mu \mathrm{eV} / \mathrm{at}\right)$, we obtain $\mathrm{N}_{/ /}=171$ and $\mathrm{N}_{\perp}=174$. We note that, as expected, $N_{/ /}$and $N_{\perp}$ are very similar. We underline that the values of $N_{/ /}$and $N_{\perp}$ allow to fit according to Eq. (1) the experimental magnetization curves recorded with angle $\theta$ ranging from the easy to the hard axis of magnetization.
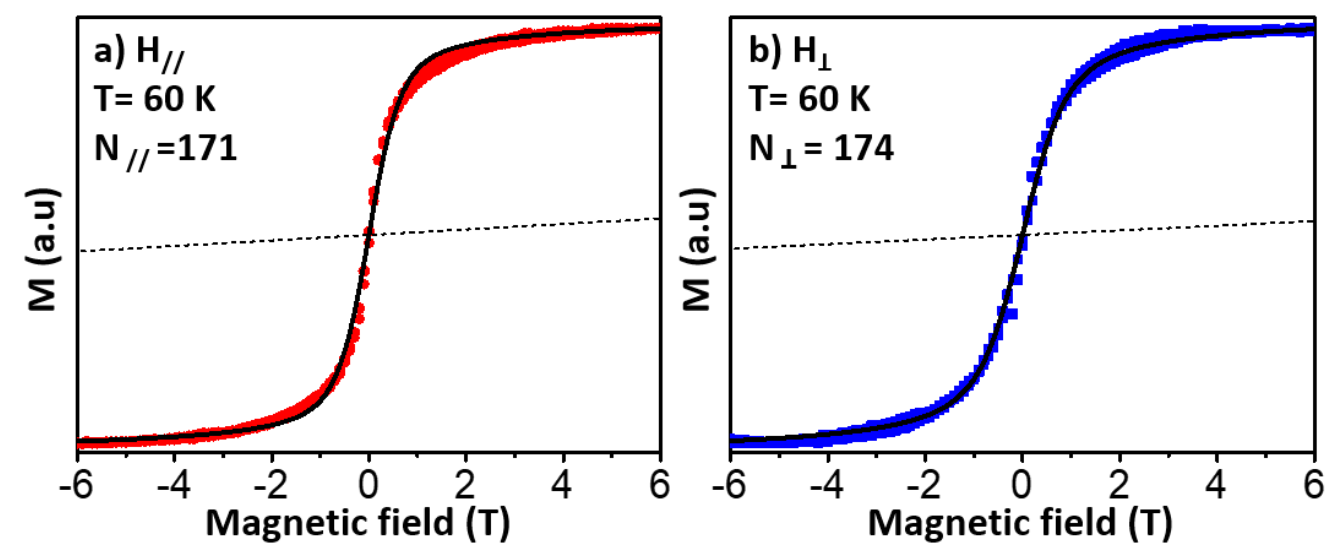

FIG. 6: Magnetization (M) of $4 \mathrm{MLco}$ recorded at $60 \mathrm{~K}$ at the $\mathrm{L}_{3} \mathrm{Co}$ edge. (a) $\mathrm{M}$ as a function of the applied field measured in the $\mathrm{H} / /$ configuration with $\theta=70^{\circ}$, normalized to its saturation value at $6 \mathrm{~T}$ (filled red dots). The solid

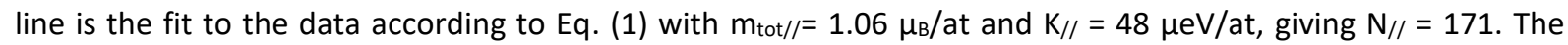
dashed line corresponds to the magnetization of an isolated Co atom, given by Eq. (1) with $\mathrm{N}=1$. (b) the same as (a) in the $\mathrm{H}_{\perp}$ configuration with $\mathrm{m}_{\mathrm{tot} \perp}=0.98 \mu_{\mathrm{B}} /$ at and $\mathrm{K}_{\perp}=51 \mu \mathrm{eV} /$ at, giving $\mathrm{N}_{\perp}=174$. Experimental magnetization $M$ is represented with filled blue squares.

The fits made at $\mathrm{T}=40 \mathrm{~K}, 60 \mathrm{~K}, 80 \mathrm{~K}$ and $100 \mathrm{~K}$ are summarized in Table I. The upper (respectively lower) values of $\mathrm{N}_{/ /}$and $\mathrm{N}_{\perp}$ are obtained by fitting the magnetization with the lower (respectively upper) values of the total magnetic moment and the MAE. We can assume that within the uncertainties $\mathrm{N}_{/ /}$and $\mathrm{N}_{\perp}$ remain constant while varying the temperature, leading to an average value of $(171 \pm 30)$ Co atoms and $(163 \pm 25)$ Co atoms, respectively. Thus, as expected, $N_{/ /}$and $N_{\perp}$ are identical within the uncertainties. A statistical analysis of STM images published in [25] revealed that, in the early stages of growth, more than $80 \%$ of the Co dimer nanolines are coupled to a second nanoline of similar length on the same Si DNRs. Thus, considering double rows of Co dimers and the two upper Co 
magnetic layers, the length of a spin block measured in the $\mathrm{H}_{/ /}$configuration corresponds to $\mathrm{N}_{/ /} / 8=(21$ $\pm 4)$ dimers i.e. $(9 \pm 2) \mathrm{nm}$ and to $\mathrm{N}_{\perp} / 8=(20 \pm 3)$ dimers i.e. $(8.5 \pm 1.5) \mathrm{nm}$ in the $\mathrm{H}_{\perp}$ configuration. We outline that from an experimental point of view, the size of the spin blocks cannot be straightforwardly compared to the length of the Co nanolines since XMCD probes the magnetic response of the whole Co ultrathin film while STM gives only access to the morphology of the top layer. In our case of a nonperfect layer-by-layer growth, we thus solely observe by STM the uncovered parts of the different Co layers. Nevertheless, the length of the spin blocks is remarkably similar to the length of the Co nanolines $(\sim 6 \mathrm{~nm})$ estimated from STM measurements. We remind that this length has been assimilated to the nanoline length of the first Co layer. It can be assumed that, during the growth, some Co nanolines in the upper layers have coalesced in the direction parallel to the Si NRs, forming longer Co nanolines. Moreover, the larger length derived from our fits could result from the fact that the contribution of the first two Co layers is not considered in the fits using Eq. (1). Even if XMCD measurements revealed that these two Co layers have a weak magnetic response, taking into account their contribution would reduce the estimated length of the spin blocks. Thus, our fit process strongly suggests that the Co system behaves as a superparamagnetic system composed of thermally fluctuating $1 \mathrm{D}$ segments.

\begin{tabular}{ccccc}
\hline \hline & $\mathrm{T}=40 \mathrm{~K}$ & $\mathrm{~T}=60 \mathrm{~K}$ & $\mathrm{~T}=80 \mathrm{~K}$ & $\mathrm{~T}=100 \mathrm{~K}$ \\
\hline $\mathrm{N}_{/ /}$ & $132<\mathbf{1 5 3}<184$ & $147<\mathbf{1 7 1}<204$ & $151<\mathbf{1 7 6}<209$ & $158<\mathbf{1 8 3}<217$ \\
$\mathrm{~N}_{\perp}$ & $143<\mathbf{1 6 4}<194$ & $152<\mathbf{1 7 4}<205$ & $139<\mathbf{1 5 9}<186$ & $134<\mathbf{1 5 3}<179$ \\
\hline \hline
\end{tabular}

Table I. Values of $\mathrm{N} / /$ and $\mathrm{N}_{\perp}$ as a function of the temperature derived from fits of the experimental magnetization of 4 MLco according to Eq. (1).

\section{CONCLUSION}

The combined STM-XMCD study reported in this paper provides a comprehensive description of the magnetic Co nanolines grown on a 1D Si nanotemplate. The Co ultrathin film, $4 \mathrm{ML}$ o thick, is composed of dimer nanolines aligned with respect to the Si NRs and are $\sim 6 \mathrm{~nm}$ in length. Fielddependent magnetization measurements evidence that an in-plane magnetization is favored. The Co system behaves as a superparamagnetic system composed of thermally fluctuating 1D segments (spin blocks) of two layers thick, containing each $\sim 170$ coupled Co atoms. The length of the spin block, $\sim 9$ $\mathrm{nm}$, is found to be similar to the length of the grown Co nanolines. This length is didacted by the Co diffusion into the Si layer. It would appear interesting to grow longer nanolines to known whether the ferromagnetic coupling of magnetic atoms is limited by the length of the nanolines or is related to the 
occurrence of critical fluctuations. Finally, we stress that our study illustrates the richness of the magnetic anisotropy of low dimensional systems.

\section{ACKNOWLEDGMENTS}

Support from the DEIMOS beamline staff at SOLEIL is greatly acknowledged. The authors gratefully thank Andrés Saúl and Philippe Ohresser for fruitful discussions.

[1] P. Gambardella, S. Rusponi, M. Veronese, S. S. Dhesi, C. Grazioli, A. Dallmeyer, I. Cabria, R. Zeller, P. H. Dederichs, K. Kern, C. Carbone, and H. Brune, Science (80-. ). 300, 1130 LP (2003).

[2] S. Rusponi, T. Cren, N. Weiss, M. Epple, P. Buluschek, L. Claude, and H. Brune, Nat. Mater. 2, 546 (2003).

[3] I. G. Rau, S. Baumann, S. Rusponi, F. Donati, S. Stepanow, L. Gragnaniello, J. Dreiser, C. Piamonteze, F. Nolting, S. Gangopadhyay, O. R. Albertini, R. M. Macfarlane, C. P. Lutz, B. A. Jones, P. Gambardella, A. J. Heinrich, and H. Brune, Science (80-. ). 344, 988 LP (2014).

[4] F. Donati, S. Rusponi, S. Stepanow, C. Wäckerlin, A. Singha, L. Persichetti, R. Baltic, K. Diller, F. Patthey, E. Fernandes, J. Dreiser, Ž. Šljivančanin, K. Kummer, C. Nistor, P. Gambardella, and H. Brune, Science (80-. ). 352, 318 LP (2016).

[5] P. Gambardella, A. Dallmeyer, K. Maiti, M. C. Malagoli, W. Eberhardt, K. Kern, and C. Carbone, Nature 416, 301 (2002).

[6] J. Dorantes-Dávila and G. M. Pastor, Phys. Rev. Lett. 81, 208 (1998).

[7] R. Félix-Medina, J. Dorantes-Dávila, and G. M. Pastor, New J. Phys. 4, 100 (2002).

[8] B. Lazarovits, L. Szunyogh, P. Weinberger, and B. Újfalussy, Phys. Rev. B 68, 24433 (2003).

[9] B. Lazarovits, L. Szunyogh, and P. Weinberger, Phys. Rev. B 67, 24415 (2003).

[10] F. D. C. de Lima and R. H. Miwa, Mater. Chem. Phys. 194, 118 (2017).

[11] J. Honolka, T. Y. Lee, K. Kuhnke, D. Repetto, V. Sessi, P. Wahl, A. Buchsbaum, P. Varga, S. Gardonio, C. Carbone, S. R. Krishnakumar, P. Gambardella, M. Komelj, R. Singer, M. Fähnle, K. Fauth, G. Schütz, A. Enders, and K. Kern, Phys. Rev. B 79, 104430 (2009).

[12] B. Dupé, J. E. Bickel, Y. Mokrousov, F. Otte, K. von Bergmann, A. Kubetzka, S. Heinze, and R. Wiesendanger, New J. Phys. 17, 23014 (2015).

[13] P. Gambardella, A. Dallmeyer, K. Maiti, M. C. Malagoli, S. Rusponi, P. Ohresser, W. Eberhardt, C. Carbone, and K. Kern, Phys. Rev. Lett. 93, 77203 (2004).

[14] J. Hong and R. Q. Wu, Phys. Rev. B 67, 20406 (2003).

[15] M. Menzel, Y. Mokrousov, R. Wieser, J. E. Bickel, E. Vedmedenko, S. Blügel, S. Heinze, K. von Bergmann, A. Kubetzka, and R. Wiesendanger, Phys. Rev. Lett. 108, 197204 (2012).

[16] L. Michez, K. Chen, F. Cheynis, F. Leroy, A. Ranguis, H. Jamgotchian, M. Hanbücken, and L. 
Masson, Beilstein J. Nanotechnol. 6, 777 (2015).

[17] D. Nečas and P. Klapetek, "Gwyddion: an open-source software for SPM data analysis," Cent. Eur. J. Phys., 10, 181 (2012).

[18] P. Ohresser, E. Otero, F. Choueikani, K. Chen, S. Stanescu, F. Deschamps, T. Moreno, F. Polack, B. Lagarde, J.-P. Daguerre, F. Marteau, F. Scheurer, L. Joly, J.-P. Kappler, B. Muller, O. Bunau, and P. Sainctavit, Rev. Sci. Instrum. 85, 13106 (2014).

[19] L. Joly, E. Otero, F. Choueikani, F. Marteau, L. Chapuis, J. Synchrotron Rad. 21, 502 (2014).

[20] G. Prévot, C. Hogan, T. Leoni, R. Bernard, E. Moyen, and L. Masson, Phys. Rev. Lett. 117, 276102 (2016).

[21] M. Daher Mansour, R. Parret, and L. Masson, J. Vac. Sci. Technol. A 36, 61402 (2018).

[22] J. I. Cerdá, J. Sławińska, G. Le Lay, A. C. Marele, J. M. Gómez-Rodríguez, and M. E. Dávila, Nat. Commun. 7, 13076 (2016).

[23] F. Ronci, G. Serrano, P. Gori, A. Cricenti, and S. Colonna, Phys. Rev. B 89, 115437 (2014).

[24] H. Sahaf, C. Léandri, E. Moyen, M. Macé, L. Masson, and M. Hanbücken, EPL (Europhysics Lett. 86, 28006 (2009).

[25] F. Dettoni, H. Sahaf, E. Moyen, L. Masson, and M. Hanbücken, EPL (Europhysics Lett. 94, 28007 (2011).

[26] P. Ohresser, G. Ghiringhelli, O. Tjernberg, N. B. Brookes, and M. Finazzi, Phys. Rev. B 62, 5803 (2000).

[27] W. F. Pong, Y. K. Chang, R. A. Mayanovic, G. H. Ho, H. J. Lin, S. H. Ko, P. K. Tseng, C. T. Chen, A. Hiraya, and M. Watanabe, Phys. Rev. B 53, 16510 (1996).

[28] B. T. Thole, P. Carra, F. Sette, and G. van der Laan, Phys. Rev. Lett. 68, 1943 (1992).

[29] P. Carra, B. T. Thole, M. Altarelli, and X. Wang, Phys. Rev. Lett. 70, 694 (1993).

[30] C. T. Chen, Y. U. Idzerda, H.-J. Lin, N. V Smith, G. Meigs, E. Chaban, G. H. Ho, E. Pellegrin, and F. Sette, Phys. Rev. Lett. 75, 152 (1995).

[31] R. Wu, D. Wang, and A. J. Freeman, Phys. Rev. Lett. 71, 3581 (1993).

[32] G. Y. Guo, H. Ebert, W. M. Temmerman, and P. J. Durham, Phys. Rev. B 50, 3861 (1994).

[33] A. Lehnert, S. Dennler, S. Rusponi, M. Etzkorn, G. Moulas, P. Bencok, P. Gambardella, H. Brune, and J. Hafner, Phys. Rev. B 82, 94409 (2010). 